\title{
Ethnische Konflikte in den Staaten der Dritten Welt
}

\author{
Von Jakob Rösel
}

\section{Vorwort}

Die folgende Betrachtung geht den Entstehungsbedingungen und Formen ethnischer Konflikte nach. Obwohl sie diese ethnischen Konflikte maßgeblich im Rahmen der nachkolonialen, modemisierenden Staaten der Dritten Welt betrachtet, tritt sie manchmal aus diesem Rahmen heraus. Voraussetzung, Rahmenbedingung oder Auslöser ethnischer Konflikte - ethnische Identität, der bürokratische Territorialstaat oder der Modemisierungsprozeß - weisen über diesen Rahmen hinaus. Nach einer provisorischen Umschreibung ethnischer Gruppen und ethnischer Identitäten soll die Entstehung ethnischer Konflikte anschließend die Politisierung dieser Konflikte und das Resultat dieser Politisierung betrachtet werden. Diese Abfolge wurde gewählt, um diese drei Schritte unterscheidbar zu machen und um diesen Prozessen den Anschein der Selbstverständlichkeit zu nehmen.

\section{Einleitung: Ethnische Gruppen}

Ethnische Gruppen wollen wir als von zwei Merkmalen oder besser zwei sozialen Prozessen als gekennzeichnet ansehen.

1. Ethnische Gruppen sind selbstdefinierte Gruppen. Sie sind nicht zwingend in einer offensichtlichen räumlichen Geschiedenheit vorfindbar, sie sind nicht zwingend zur eigenständigen biologischen Reproduktion fähig und sie verfügen auch nicht immer über eine vollständige wirtschaftliche, politische oder religiöse Eigenständigkeit.1 Ethnische Gruppen

1 Barth, F. (ed.), Ethnic Groups and Boundaries, The Social Organization of Culture Difference, London 1969, Introduction F. Barth, Seite 11 "(The) ideal type definition (of an ethnic group) is not so far removed in content from the traditional proposition that a race $=\mathbf{a}$ culture $=\mathrm{a}$ language and that a society $=\mathbf{a}$ unit which rejects or discriminates against others... My quarrel is not so much with the substance of these characteristics... my main objection is that such a formulation prevents us from understanding the phenomenon of ethnic groups and their place in human society and culture. This is because it begs all the critical questions: While purporting to give an ideal type model of a recurring empirical form it implies a preconceived view of what are the significant factors in the genesis, structure and function of such groups. Most critically, it allows us to assume that boundary maintenance is unproblematical and follows from the isolation which the itemized characteristics imply: Racial difference, cultural difference, social separation and language barriers, spontaneous and organized enmity... We are led to imagine each group developing its 
beruhen stattdessen auf einem sozialen Prozeß der Selbstdefinition, der Abgrenzung. Sie definieren sich auf der Grundlage von sozialen oder biologischen Gemeinsamkeiten, die sich aus so verschiedenen biologischen und sozialen Bestimmungsdimensionen wie der "Rasse", der Sprache, der Religion, des Verhaltens, der Institutionen, der historischen Erfahrungen, des Siedlungs- oder Herkunftsgebiets ableiten lassen. Diese Definition, diese Selbst- oder Fremdfestlegung erfolgt zumeist unreflektiert und seit Generationen. Sie bildet - in einem nicht mehr bestimmbaren Kreislauf - beobachtbare Gemeinsamkeiten ab und verstärkt sie und setzt sie verbindlich. Vorgabe und soziale Produktivität und Plastizität lassen sich nur noch selten auseinanderhalten. Die begründete oder unbegründete Annahme weitgehend unreflektierter, kollektiver und dauerhafter Festlegung ethnischer Identität verbietet aber nicht eine gegenläufige Annahme: Die Annahme, daß ethnische Identität nicht auch von Einzelnen kalkuliert und reflektiert verändert oder modifiziert wird.

Auch wenn bei einer Vielzahl ethnischer Gruppen einzelnen dieser Bestimmungsdimensionen stets eine besondere Bedeutung zukommt, so hindert uns dennoch nichts an der Annahme, daß dieser Liste von Bestimmungsdimensionen im Einzelfall weitere hinzugefügt werden können, zwingt uns die Erfahrung zur Einsicht, daß keine der genannten Dimensionen für die Bestimmung ethnischer Identität unverzichtbar bleibt. Der soziale Prozeß der Selbstdefinition beruht damit auf einer relativen Beliebigkeit der angesprochenen, also selektierten Dimensionen der sozialen oder biologischen Gemeinsamkeit. Dabei erscheint es allerdings plausibel, daß die jeweils postulierte ethnische Identität in dem Maße anschaulicher und überzeugender erscheint, indem sie auf Gemeinsamkeiten verweist, die zweifelsfrei beobachtbar werden und indem sie auf Gemeinsamkeiten in einer Vielzahl von verschiedenen Vergleichsdimensionen ruht. Dem Vorteil einer solchen Vielzahl sozialer oder biologischer Gemeinsamkeiten steht aber oft das Problem gegenüber, diese so verschiedenen unterstellten oder nachweisbaren Gemeinsamkeiten auch wechselseitig zur Deckung zu bringen.

Der Akt der sozialen Selbstdefinition verlangt damit die Fähigkeit des Vergleichs und der Unterscheidung, also die Bestimmung von Gemeinsamkeiten, die Fähigkeit zu einer Wahl, welcher Dimension der Vergleichbarkeit besondere Aufmerksamkeit geschenkt werden muß, und die Fähigkeit, eine angemessene Deckungsgleichheit zwischen diesen verschiedenen Gemeinsamkeiten zu erzielen.

Der Akt der sozialen Selbstdefinition bleibt aber letztendlich beliebig: Eine Vielzahl von Dimensionen steht zur Begründung ethnischer Identität zur Verfügung, eine jeweils

cultural and social form in relative isolation, mainly in response to local ecological factors, through a history of adaption by invention and selective borrowing. The history has produced a world of separate people, each with their culture and each organized in a society which can legitimately be isolated for description as an island to itself." 
beanspruchte ethnische Identität beginnt auf beliebigen Grundlagen zu ruhen. ${ }^{2}$ Dieser Beliebigkeit der Grundlagen der ethnischen Identität steht allerdings eine konstante Form ihrer Einschätzung, ihrer Interpretation gegenüber.

2. Die jeweiligen Gemeinsamkeiten gelten nicht als individuell und durch einen Akt freier Wahl erworben, sie gelten als ererbt, als von einem Kollektiv zugeschrieben, jenem Kollektiv, das sich durch diese Gemeinsamkeiten von anderen abgrenzt. Dies ist im Falle biologischer Merkmale trivial, im Falle sozialer Merkmale weit weniger. Hier relativiert, verdrängt oder schwächt die Vorstellung, diese sozialen Merkmale seien ererbt, alle Hinweise, Wahrscheinlichkeiten und Ansprüche, diese Merkmale könnten auch über den Weg individueller Wahl übernommen oder zugänglich werden. Zugleich wird der askriptive, der ererbte Charakter dieser Merkmale in die Vergangenheit, oft bis zum Beginn der Geschichte oder einem mythischen Datum zurückverlängert. Die konstitutiven Gemeinsamkeiten sind nicht nur ererbt, zugeschrieben, sie wurden schon immer ererbt. Sie sind damit Bestandteil einer ursprünglichen Identität der Gruppe, Bestandteil einer allerersten Ordnung, sie sind primordial. Diese Gemeinsamkeiten beanspruchen damit eine doppelte, eine zeitliche wie eine soziale Vorrangstellung.

Ưber den Weg der Rückverlängerung des Zuschreibungsprozesses bis zum Beginn einer allgemeinen oder der jeweiligen ethnischen Geschichte verwandeln sich deshalb die nachweisbaren oder unterstellten Gemeinsamkeiten - der Sprache, der Religion, des Siedlungsoder Herkunftgebiets etc. - zum partiellen Nachweis der Existenz einer umfassenden Abstammungs- und historischen Lebensgemeinschaft.

Die gewählte Umschreibung ethnischer Gruppen hat den Vorteil, daß sie den Akzent der Beschreibung von augenfälligen, aber nicht immer vorfindbaren Merkmalen einer räumlichen, biologischen oder sozialen Eigenständigkeit der Gruppe auf subjektive Prozesse der Grenzziehung, der Absonderung, der Differenzierung verschiebt. Sie ist damit auch in Situationen anwendbar, in denen ethnische Gruppen in übergreifenden politischen, wirtschaftlichen und religiösen Herrschafts- und Austauschsystemen, in Systemen "polyethi-

2 "The features that are taken into account are not the sum of 'objective' differences, but only those which the actors themselves regard as significant. Not only do ecological variations mark and exaggerate differences, some cultural features are used by the actors as signals and emblems of differences, others are ignored and in some relationships radical differences are played down and denied... The critical focus of investigation from thes point of view becomes the ethnic boundary that defines the group, not the cultural stuff that it encloses social boundaries to which we must give our attention are of course social boundaries, though they may have territorial counterparts...", Barth, F. (ed.), Ethnic Groups... a.a.O., S.14. 
scher Inkorporation" stehen. ${ }^{3}$ Dies trifft häufig für koloniale aber auch noch nachkoloniale Staaten zu. Eine solche Umschreibung erweist sich deshalb auch gegenüber komplexen traditionalen, sich modemisierenden oder modernen - Gesellschaften als anwendbar. Der Vorteil ihrer Universalität bedingt zugleich den doppelten Nachteil mangelnder Spezifizität. Es wird schwer, auf der Grundlage dieser Umschreibung ethnische Gruppen von anderen Gruppenorganisationen wie der Kaste oder dem Stand abzugrenzen. Und es fällt schwer, die besondere Form ethnischer Gruppen zu charakterisieren, die sich im Rahmen des Modemisierungsprozesses einstellt.

Diese ethnischen Gruppen sind nicht mehr nur charakterisiert durch die Praxis einer traditionellen Lebensform und damit über die Verfügung selbstverständlicher, nicht weiter reflektierter sozialer oder biologischer Gemeinsamkeiten, stattdessen verwandelt sich der Verweis auf die Gemeinsamkeiten einer traditionellen Lebenspraxis zu einem wissenschaftlichen Projekt, zu einer wissenschaftlichen Anstrengung. Der Historiker, der Philologe, der Volks- und Völkerkundler, der Geograph unternimmt die Arbeit einer wissenschaftlichen Begründung, Systematisierung und schließlich Wiederherstellung der Ursprünglichkeit und der Reinheit dieser konstitutiven Gemeinsamkeiten. An die Stelle einer fraglosen Lebenspraxis oder des Verweises auf sakrale Begründungen - eines Ursprungsmythos, einer Einwanderungslegendem, einer fiktiven Genealogie - tritt die wissenschaftliche Begründung der Eigenart und Primordialität der jeweiligen Gruppe. Diese Anstrengung bildet zugleich eine Antwort auf die Bedrohung und die Chance, die von einem neuen Gesellschaftsentwurf ausgeht.

Ethnische Gruppen erscheinen als ein schutzbedürftiger Wert, ethnische Gruppenzugehörigkeit als soziale und kulturelle Tugend. Diese neue Schutzbedürftigkeit wäre im Rahmen einer ausschließlich ethnisch verfaßten Gesellschaft, also einer Gesellschaft, in der die Existenz eines Individuums losgelöst von einer - nicht zwingend seiner - ethnischen Gruppe entweder unvorstellbar oder widernatürlich erschienen wäre, höchst ungewöhnlich gewesen. Ethnische Gruppenzugehörigkeit wäre hier als selbstverständlich, als Bestandteil der sozialen Ordnung erschienen. Eine besondere Wertschätzung kann der ethnischen Gruppe nur entgegengebracht werden, wenn alternative soziale Existenzweisen denkbar werdenund wenn zugleichdie ethnische Gruppenzugehörigkeit als eine absolute, künftig verschollene soziale Existenzweise erscheint. Diese doppelte Alternative eine Lebens außerhalb und ohne ethnische Gruppen wird aber nur im Rahmen moderner oder sich modemisierender Gesellschaften denkbar. 4

3 J.S. Furnivall hat solche Gesellschaften am Beispiel Burmas und Javas beschrieben. Furnivall, J.S., Colonial Policy and Practice, A Comparative Study of Burma and Netherland India, New York 1956, S.303-311, "The Plural Society".

4 "On peut désormais se passer des anciennes façons de trouver son identité au sein d'une société, un homme sans famille, sans domicile fixe et sans appartenance religieuse peut mener une vie suffisamment remplie. Mais sans Etat, il n'est rien... Ce ne fut pas toujours le cas. Il y eut des 
Der modeme Territorialstaat und der Modemisierungsprozeß stellen deshalb zwei Merkmale zur Verfügung, durch die sich zeitgenössische ethnische Gruppen von ethnischen Gruppen in traditionalen Gesellschaften provisorisch unterscheiden lassen: Sie machen eine spezifische Wertschätzung und eine neue, eine theoretische Begründbarkeit ethnischer Identität möglich. Von beidem, dem modemen Territorialstaat und dem Modernisierungsprozeß geht zugleich eine Möglichkeit und eine Wahrscheinlichkeit ethnischer Konflikte aus.

\section{Die Entstehung ethnischer Konflikte}

\section{A. Der postkoloniale Staat}

Der modeme Territorial- und Verfassungsstaat hat sich heute umfassend geographisch wie politisch, horizontal wie vertikal durchgesetzt. Er ist die politische Lebensform "a la mode".5 In seinem Binnenraum hat er alle früher verbindlichen, oft weitgehend eigenständigen Sozialordnungen und Lebensformen - des erweiterten Familienverbandes, des Lokalverbandes, der religiösen Kongregation, der ethnischen Gruppen - überlagert und überformt. Er hat sie verändert aber nicht ersetzt. Seine äußere, räumliche Gestalt hat sich zugleich vollständig horizontal durchgesetzt: Die Grenzen des einen Territorialstaates sind kontingent mit denjenigen seiner Nachbarn. Nicht die Grenze, allenfalls ihr Verlauf ist strittig.

Eine Vielzahl dieser modernen Territorialstaaten, vor allem die Staaten der Dritten Welt, ruhen aber auf multiethnischen, komplexen und noch weitgehend traditionalen Gesellschaften. Wie wir aber bereits sahen, sind ehmische Gruppen nur selten als eindeutig geschiedene, primordiale Gebilde vorfindbar. Sie definieren sich selbst, sie grenzen sich selbst ab. Sie prätendieren eine Primordialität aber sie verfügen nicht zwingend darüber. Der Prozeß der Selbstdefinition ethnischer Gruppen unterliegt zugleich keiner zweifelsfreien, objektiven Verfahrensweise. Er kann damit beständig erneuert, erweitert und verändert werden. Diese Chance der sozialen Abgrenzung und Selbstdefinition zwingt uns deshalb zu der Annahme einer potentiellen und veränderbaren Multiethnizität aller dieser Staaten.

époques qui, à l'echelle de l'histoire, ne sont pas bien reculées, où l'Etat n'existe pas, sans que personne trouvât à redire à cette absence. En ce temps-là, un homme qui n'avait ni famille, ni seigneur et n'appartenait ni à une communauté locale ni à un groupe religieux majoritaire, ne parvenait à survivre qu'en devenant domestique ou esclave." Strayer, J., Les Origines Médiévales de L'Etat Modeme, Paris 1979, S.13-14.

Strayer, J., Les Origines... a.a.0., S.13-15. 
Diese faktische und potentielle Multiethnizität kann aber dem modernen Territorialstaat, im Gegensatz zu den früheren Vielvölkerimperien nicht gleichgültig bleiben. Der moderne Territorialstaat ist auch Verfassungsstaat, er ist nicht nur eine funktionale, bürokratische, er ist auch eine moralische Konstruktion.

Der moderne Territorialstaat sieht sich als demokratisch verfaßter Nationalstaat. Er erhebt also zwei Ansprüche, den Anspruch der demokratischen Verfaßtheit und den Anspruch der Nation. Auch die meisten nachkolonialen Staaten der Dritten Welt pflegen diese beiden Illusionen. Sie geben deshalb nicht nur vor, auf dem Selbstbestimmungsrecht eines Einzelnen zu ruhen, vor allem bleiben sie auf die Beantwortung der Frage zurückgeworfen, welches Kollektiv Einzelner gerade in diesem Falle berechtigt war, sich zu einem Staat zusammenzuschließen, ihm seine räumliche, territoriale Gestalt zu geben.6 Die politisch befriedigendste, wenn auch nicht überzeugendste Antwort auf diese Frage hat der Begriff der Nation geboten. Demnach sind diejenigen Einzelwesen, die seit Generationen über eine gemeinsame Region, Kultur, Sprache, Religion, politische Eigen- oder Selbständigkeit verfügt haben, zur staatlichen Selbstbestimmung befähigt und berechtigt. Neben die Bestimmung des individuellen Rechts auf Selbstbestimmung, neben die politische Erbschaft eine John Locke ist damit die Bestimmung eines kollektiven Rechts auf Selbstbestimmung, die politische, "nationalistische" Erbschaft eines Joseph Mazzini getreten.7

6 "... No regime, given mass communication, can escape making the attempt to be, or at least appear to be, democratic; and a movement for democracy must relate itself to a state actual or potential. Yet the tenets of democracy are universal in their application: 'All men are equal...'The only theory which reconciles the universalism of the democratic ideal with the actual fragmentation of democracy is nationalism. In a divided world, the idea of the people is naturally accompanied by the idea or the nation." Beales, DED., Mazzini and Revolutionary Nationalism, S.153, in: Thomson, D. (ed.), Political Ideas, Harmonsworth 1986.

7 Mahatma Gandhi hat 1905 die folgende Beschreibung Joseph Mazzinis gegeben: "Italy as a nation came into existence recently. Before 1870 Italy comprised a number of small principalities, each with its petty chief. Before 1870 , she was like the India... of today.Though the people spoke the same language and hat the same character, they all owed allegiance to different petty states. Today Italy is an independent country and her people are regarded as a distinct nation. All this can be said to be the achievement of one man. And his name - Joseph Mazzini. Joseph Mazzini was bom in Genoa on June 22, 1805. He was a man of such sterling character, so good-natured and so patriotic, that great preparations are being made throughout Europe to commemorate the centenary of his birth. For, although he dedicated his whole life to the service of Italy, he was so broadminded that he could be regarded a citizen of every country. It was his constant yeaming that every nation should become great and live in unity. Even at the early age of thirteen Mazzini showed great intelligence. In spite of great scholarship that he evidenced, he gave up his books out of patriotism and untertook the study of law, and began using his legal knowledge gratuitously to help the poor. Then he joined a secret organization which was working for the unification of Italy. When the Italian chiefs leamt of this, they put him in prison. While still in prison, he continued to advance his plans for freeing his country. At last he had to leave Italy... Though obliged to fly from place to place he did not lose heart and kept on sending his writings secretly to Italy which gradually influenced the minds of the people. He suffered a lot in the process. He had to run about in disguise to 
Diese Begründung staatlicher Selbstbestimmung, diese Grundlage staatlicher Legitimität kann sich aber gegen ihn selbst richten. Die Begründung wird dem Staat vor allem dann gefährlich, wenn er das Resultat kolonialer und oft zufälliger Grenzziehung ist und ihm in seinem Binnenraum eine große Zahl eigenständiger ethnischer Gruppen entgegensteht. Nichts hindert diese Gruppen daran, unter Verweis auf ihre sprachliche, soziale, regionale, religiöse Gemeinsamkeit, unter Verweis auf ihre Tradition politischer Eigenständigkeit, kulturelle und politische Autonomie oder sogar einen eigenen Staat zu fordem. Damit verwandelt sich dieser demokratische und nationale Legitimitätszwang, der über den Weg der demokratischen und nationalen Interesseneinbindung maßgeblich zu der Machtfülle des modemen Staates beigetragen hat, zugleich zu einer ihm spezifischen Bedrohung. Diese ethnischen Forderungen bedrohen damit die Legitimität und die Einheit des nachkolonialen Staates. In dem Maße, in dem die demokratische Verfaßtheit dieser Staaten oft wenig mehr ist als eine fromme Illusion, ist der Anspruch der Nation die einzige Legitimitätsgrundlage, über die politische Eliten oder Machthaber verfügen. Der nachkoloniale Staat kann diese Forderung ethnischer Gruppen zwar blutig aber nicht legitim unterdrücken. Da ihm aus dieser Forderung immense ideologische, politische und finanzielle Kosten erwachsen können, sieht er sich zu einer präventiven Aufgabe genötigt: zur Arbeit des "nationbuilding". Neben die bürokratische Durchsetzung des Territorialstaates tritt seine ideologische: der kollektive Geltungsanspruch der Nation. Diese Arbeit des "nation-building" soll seinem Legitimitätsanspruch zumindest nachträglich Beweiskraft verschaffen und andere daran hindern, diesen Anspruch gegen ihn zu wenden.

Damit bleibt der nachkoloniale Staat aber einem spezifischen Spannungszustand ausgesetzt. Dieser Spannungszustand läßt sich mit dem Bild eines Wettlaufs anschaulich machen. Der nachkoloniale Staat muß sich in eine Nation wandeln, bevor ethnische Gruppen sich als "Nationen" formieren, um auf dieser Grundlage ihren eigenen Staat zu fordem. Diesen Wettlauf kann der Staat aber nicht endgültig und irreversibel gewinnen. Die Umschreibung ethnischer Gruppen als selbstdefinierte und damit modifizierbare Gruppen hat bereits ein Moment der Subjektivität und des Kalküls bewußt offengehalten. Modeme und modemisierende Gesellschaften bieten genügend Anschauungsmaterial für Fälle, in denen in scheinbar homogenen Nationalstaaten fiktive oder vorfindbare, vergessene oder unterdrückte ethnische Identitäten neu belebt werden konnten. Die Aufgabe des "nation-building" hebt damit die Möglichkeit einer Bedrohung durch ethnische Forderungen nicht auf. Von den politischen Forderungen aber, die ethnische Gruppen erheben können und die der nachkoloniale Staat nie vollständig verhindern kann, geht eine besondere Bedrohung aus. Sie ziehen die

evade spies. Even his life was frequently in danger, but he did not care... In 1848 Mazzini retumed... to Italy, and set up the self-goveming State of Italy. It did not last long, thanks to the activities of crafty persons. But, though Mazzini had to flee the country once again, his influence did not fade. The seed of unity that he had sown endured and, though Mazzini remained in banishment, Italy became a single united kingdom in 1870..." The Collected Works of Mahatma Gandhi, Vol. V, 1905-1906, S.27-28. 
Funktionsfähigkeit, die Gestalt und im Grenzfall die Einheit des Staats in Zweifel. Die immensen Kosten, die dem nachkolonialen Staat aus solchen Autonomieforderungen schließlich erwachsen, können ihn deshalb geneigt machen, begrenzten Forderungen ethnischer Gruppen nachzugeben, ethnische Gruppen und ihre Führer aufzuwerten oder bereitwilliger abzuspeisen. Nachdem er ethnische Forderungen nicht verhindern konnte, ist der Staat schließlich bereit, für deren Entschärfung, für eine politische Selbstbeschränkung der Gruppen zu bezahlen. Die spezifische, historisch neuwertige Verletzbarkeit des Nationalstaates gegenüber ethnischen "nationalistischen" Forderungen begründet damit auch politische Interessen und ein Kalkül, ihm gegenüber die "ethnische Karte" auszuspielen.8 Stimmenschwache Minderheitsgruppen, aber auch politischschwache Mehrheitsgruppen können versuchen, ihre Schwäche auf der Grundlage einer solchen expliziten oder impliziten Bedrohung zu überwinden.

Der nachkoloniale Staat steht damit oft ethnischen Gruppen gegenüber, er ist durch politische Forderungen dieser Gruppen spezifisch und nachhaltig verwundbar und diese Verwundbarkeit lädt ethnische Gruppen und ihre Führer dazu ein, diese zu nutzen. Der nachkoloniale Staat macht damit ethnische Konflikte möglich und denkbar. Was macht sie wahrscheinlich.

\section{B. Der Modernisierungsproze $\beta$}

Der Begriff der Modemisierung ist wenig befriedigend, aber zugleich unumgänglich, wenn in der gebotenen Unverbindlichkeit die Vielfalt jener sozialen, politischen, technischen und kulturellen Wandlungen und Herausforderungen umschrieben werden soll, denen die koloniale Welt und in verstärktem Maße die nachkoloniale Welt, ihre ethnischen Gruppen

8 "Ethnicity is a plastic, variegated, and originally ascriptive trait, that, in certain historical and social economic circurnstances, is readily politicized. Such fertile circumstances abound in modem and transitional (modemizing) societies... Such societies... generate political entrepreneurs with a conscious and realistic interest in mobilizing ethnicity from a psychologicalor cultural or social datum into political leverage for the purpose of altering or reinforcing... systems of structured inequality between and among ethnic groups... As a result, in modem and transitional societies... politicized ethnicity has become the crucial principle of political legitimation and delegitimation of systems, states, regimes and governments and at the same time has also become an effective instrument for pressing mundane interests in society's competition for power, status and wealth... The political genius of ethnicity in the contemporary world... lies... in its ability to combine emotional sustenance with calculated strategy. A circular process has set in: As governments become increasingly sensitive to ethnopolitical demands, ethnic groups are thereby given an incentive to organize themselves into cohesive interest groups for the extraction of desired policies and outputs, and as this ethnic consolidation proceeds, governments render themselves yet more responsive to it. Thus ethnicity becomes both instrumental and affective...", Rothschild, J., Ethnopolitics, A Conceptual Framework, New York 1981, S.1-2, 61. 
und traditionellen Institutionen ausgesetzt sind. Der Begriff umfaßt soviel, daß er an einen Kreis erinnert, dessen Mittelpunkt überall und dessen Grenzen nirgendwo zu bestimmen sind. Da er somit der Beliebigkeit ausgesetzt bleibt, soll er hier folgendermaßen provisorisch umschrieben werden. Als Modemisierungsprozeß soll uns die wachsende globale Durchsetzung und Anerkennung universal gültiger oder anwendungsfähiger Stile, Werte, Normen, Erkenntnisse und Techniken gelten. Der moderne Territorial- und Verfassungsstaat - Bestandteil, Träger und Wirkung des Modemisierungsprozesses - bietet eine Anschauung dieser Umschreibung: Er ist gegenwärtig die Staatsform "a la mode", er gilt als legitim, üblich, als vemünftig und er ist unverzichtbar.

Mit der Durchsetzung dieses Territorialstaats und dem abhängig oder unabhängig von ihm einsetzenden Wandlungsprozeß verband sich bis vor kurzem die Annahme, ethnische Gruppen- und Identitätsmuster würden auf Dauer geschwächt und durch neue Organisationsmodelle und Identitätsmuster ersetzt. Die Erfahrung der Gegenwart, vor allem diejenige der Staaten der Dritten Welt, hat allerdings gezeigt, da $ß$ der Modernisierungsproze $\beta$ ethnische Identitätsmuster zwar umfassend verändert, aber nicht überwindet. Stattdessen macht der Modemisierungsprozeß einen Erhalt, eine Rekonstruktion, eine Reflexion und eine Idealisierung ethnischer Identität möglich und er löst ethnische Konflikte in einer neuen Gestalt und in einer neuen Intensität aus. Im Maßstabe der Durchsetzung des Territorialstaates und eines beschleunigten Modernisierungsprozesses werden traditionellen, ethnischen Identitätsmustem, traditionellen Wirtschafts- und Herrschaftsformen und traditionellen Orientierungs- und Einschätzungsmustern neue entgegengestellt. Diese neuen Identitätsmuster, Strukturen und Erwartungen treten vorerst neben und nicht an die Stelle dieser traditionellen Ordnungen und von dieser Altemative geht eine Herausforderung, eine Chance oder ein Bedrohung, immer eine Verwandlung ethnischer Muster aus.

\section{Die neuen Identitätsmuster}

Prozesse wachsender Zentralisierung und Modemisierung schwächen oder relativieren in immer stärkerem Umfange jene Merkmale - der Religion, der Sprache, der politischen und sozialen Eigenständigkeit, des Siedlungsgebiets - auf deren Grundlage ethnische Gruppen sich traditionell definieren.

Im Rahmen eines sạkkularen Staatswesens verwandelt sich die religiöse Zugehörigkeit von einer kollektiven Verbindlichkeit zu einer Frage der individuellen Wahl. Die Durchsetzung einer neuen einheitlichen Staatssprache und einer oft synthetischen, neuen nationalen Kultur marginalisiert die Sprache und die kulturellen Traditionen von Minderheitsgruppen. Der Zentralismus einer bürokratischen Verwaltung schwächt Traditionen einer politischen und sczialen Eigenständigkeit. Urbanisierung und Wanderungsbewegungen verlagem, verändern oder zerstören alte ethnische Siedlungsgebiete. Neue gesamtstaatliche, "natio- 
nale" oder metropolitane Verhaltensstile und Moden, verdrängen die traditionellen Verhaltensstile und konservieren sie als museale Folklore. An die Stelle der offensichtlichen Gemeinsamkeit einer Gruppe oder einer Region tritt diejenige des Zeitabschnitts, der Mode. Die religiösen und volkstümlichen Entstehungsmythen der Gruppe werden in Zweifel gezogen. Die unterstellte Gemeinsamkeit, Ausschließlichkeit und Sinnfälligkeit einer ethnischen Geschichte kann von einer neuen Geschichtsschreibung als das Zufallsprodukt von Wanderungsbewegungen, Akkulturationen und Machtprozessen relativiert und abgewertet werden. Der ethnische Geschichtsentwurf wird von einer nationalistischen Geschichtsschreibung verdrängt oder ihr subsumiert.

Alle diese Entwicklungen sind bereits während der Kolonialzeit begründet worden, sie verstärken sich nach der Unabhängigkeit. Die Relativierung oder die Schwächung ethnischer Definitionsmerkmale, die von dieser Entwicklung ausgehen, muß aber nicht zwingend als Bedrohung aufgefaßt werden. Zentralisierung und Modemisierung können auch als Fortschritt, als Befreiung, als Chance der Freizügigkeit und der wachsenden individuellen Selbstbestimmung interpretiert werden. Als Bedrohung muß diese Entwicklung erscheinen, wenn der Erschüttenung traditioneller Identitätsmuster keine Alternative entgegensteht. Man kann sich nun aber die Frage stellen, inwiefern ein anlaufender Modernisierungsprozeß neue, äquivalente und sinnfällige Identitätsmuster zur Verfügung stellen kann.

In modemisierten Gesellschaften erwirbt der Einzelne Identität über den Weg der Bildungsund Berufsqualifizierung, also des individuellen Einsatzes, der individuellen Befähigung und der individuellen Wahl. Diese Qualifikationen und Entscheidungen lassen den Einzelnen in Berufsstellungen, in Berufs-, Partei- und Kulturorganisationen eintreten, die angebbaren, empirischen und modifizierbaren Zwecken dienen. Die Sicherung dieser Funktionen' konstituiert und integriert das Gebilde einer modemisierten Gesellschaft. Identität wird damit in einem wachsenden Umfange individuell erworben, im Rahmen von Berufsstellungen und Verbandsmitgliedschaften, die funktional bestimmt sind und die in letzter Instanz die Bestands- und die Entwicklungsfähigkeit, die "Makro-Integration" einer modemisierten Gesellschaft sichern.9 Diese Identitätsmuster können und wollen dem Einzelnen keinen über seine Gegenwart hinausreichenden Stellenwert in einem umfassenden - ethnischen oder religiösen - Geschichtsplan zuschreiben. Sie verzichten darauf, dem Einzelnen - sub specie aeternitatis - einen höheren Sinn und eine höhere Zweckdienlichkeit zubilligen zu wollen. Allerdings muß offenbleiben, ob selbst in modemisierten Gesellschaften diese

9 Rothschild, J., Ethnopolitics... a.a.O., S.5, "(There) is a need for some psychological distance and social autonom from the technocratic rationality that fuels the scientific modemization process... Science, technology, material productivity, and the technocratic style of decisionmaking that they entail are today suspect... for supposedly leaving man unfulfilled on some inner spiritual frontier." 
neuen Identitätsmuster ethnische oder religiöse vollständig ersetzen oder lediglich ergänzen können.

Unter den Bedingungen eines noch fragmentarischen und ungleichen Modernisierungsprozesses setzen aber diese Identitätsmuster Entscheidungsspielräume und Organisationsstrukturen voraus, die erst im Entstehen sind. Sie erscheinen deshalb weder sinnfällig noch gleichwertig. In dieser Situation kann eine erschütterte, verlorene oder zurückgewiesene ethnische Identität in einem neuen Licht erscheinen. In den Worten des amerikanischen Dichters Robert Frost ist das Zuhause "The place where, when you come, they have to take you in." 10 Die Rekonstruktion und die Idealisierung einer ethnischen Identität stellen sich deshalb gerade im Maßstabe ihrer Erschütterung ein. Prozesse der Rekonstruktion werden möglich, die sich der Grenze der Erfindung nähern.

Eine drohende Anomie und die daraus resultierende erhoffte Rückkehr, die Rekonstruktion und die Idealisierung ethnischer Identität bilden aber eine Erfahrung und einen Erfahrungsschluß, die sich bereits vor der Unabhängigkeit, während der Kolonialzeit eingestellt haben. Die einheimische Bevölkerung, vor allem die einheimische Elite mußte die koloniale Begründung eines Territorialstaates und den Beginn wirtschaftlicher und kultureller Modemisierung als Bestandteil oder Resultat einer überlegenen Gruppenkultur, derjenigen der Kolonialherren empfinden. Ein begrenzter Teil der alten oder einer neuen Elite suchte zugleich den Zugang zu den Verwaltungsstellen und den neuen Wirtschaftschancen der Kolonialmacht. Indem diese Elite sich von ihren ethnischen Traditionen abwandte und sich einer neuen kolonialen Kultur - ihrer Sprache, ihrer Bekleidung, ihren Manierismen, ihren Urteilsmustern - annnäherte, glaubte sie sich, Anstellung und Anerkennung im Rahmen der Kolonialgesellschaft sichern zu können. Diese Anstellung und Anerkennung wurde ihr aber immer nur bis zu einer bestimmten administrativen Ebene und sozialen Grenze zuteil.11 Dieser modemisierten Elite erscheint die Kolonialkultur deshalb in besonderem Maße als überwältigend, zerstörerisch und abweisend. Sie haben ihre ethnische Identität zurückgewiesen und sehen sich nun um den Lohn dieser Anstrengung betrogen. Für diese Grenzgänger zwischen zwei Kulturen wird die Rekonstruktion und Idealisierung ethnischer Iden-

10 Frost, R., The Death of the Hired Man, in: Rothschild, J., Ethnopolitics a.a.0., S.6.

11 So schreibt der Inder Dadabhai Naoroji 1881 in einem Memorandum für den "Secretary of State for India": "The power that is now being raised by the spread of education, though yet slow and small, is one that in time must for weal or woe, exercise great influence... The thousands that are being sent out by the universities every year, find themselves in a most anomalous position. There is no place for them in their motherland. They may beg in the streets or break stones on the road, for aught the rulers seem to care for their natural rights, positions and duties in their own country... Scores of Europeans must go from (Great Britain) to take up what belongs to them... The educated find themselves simply so many dummies, omamented with the tinsel of school education, and then their whole end and aim of life is ended... The power that the rulers are, so far to their credit, raising, will, as a Nemesis, recoil against themselves." Bei Kedourie, E., (ed.), Nationalism in Asia and Africa, London 1971, S.355-356. 
tität zu einer psychologischen Notwendigkeit. Sie können und müssen mit nichts beginnen. Sie können zugleich diese Rekonstruktion leisten, weil ihre neue Bildung und ihr Kontakt mit der kolonialen Gesellschaft ihnen jene antiquarischen Interessen und jene wissenschaftlichen Methoden vermittelt haben, die bei dieser Aufgabe notwendig werden. Sie, die dank ihrer Stellung und Bildung über die Chance des Vergleichs verfügen und die Überlegenheit dieser neuen Kultur im allgemeinen wie im persönlichen so schmerzhaft gefühlt haben, sie können diese Rekonstruktion ethnischer Identität, Kultur und Geschichte auch unter einen bestimmten Plan und eine bestimmte Aussage rücken. Ihre Rekonstruktion dient zumeist dem Nachweis, daß die jeweilige ethnische Gruppe vielleicht in einer verächtlichen Gegenwart lebe, daß sie aber über eine großartige Vergangenheit und eine noch glänzendere Zukunft verfüge.12 Die Rekonstruktion und Idealisierung ethnischer Identität, die erst unter Bedingungen einer beschleunigten Zentralisierung und Modemisierung zu einem allgemeinen Gruppenbedürfnis wird, ist deshalb oft während der Kolonialzeit bereits begonnen worden. Diese erneuerte Identität, ursprünglich gegen eine Kolonialkultur ausgerichtet, beginnt sich aber nun gegen andere ethnische Gruppen zu richten und sie dient neuen, praktischen Zwecken: Sie dient nicht mehr als Stütze des verletzten Würdegefühls einer neuen Elite, sie dient der Formulierung ethnischer Ansprüche im ökonomischen und sozialen Wettbewerb und sie macht eine verstärkte Organisation der ethnischen Gruppen möglich.

\section{Die neuen Wirtschaftsstrukturen}

Der koloniale Staat übernahm oder begründete in vielen Fällen Herrschaftsgebilde, die auf einer oft prekären Inkorporation ethnischer Gruppen "into the market-place and into the state" ruhten. 13 Diese Ordnungen kollektiver, ethnischer Arbeitsteilung und politischer Eingliederung bedrohten nicht die Identität ethnischer Gruppen, sondern erhielten und garantierten sie. Die ethnischen Gruppen galten als einander vollständig fremd, als unvergleichbar und zugleich unverzichtbar. Der Bestand der Gesamtgesellschaft beruhte auf dem Erhalt dieses politischen und wirtschaftlichen Beziehungsgefüges.

12 Bereits 1910 schilderte Bipin Chandra Pal weitsichtig, wie die Hindu-Religiosität modemisiert und politisiert werden kann und wie sich diese Reinterpretation unter einen bestimmten Geschichtsplan stellen läßt. Kedourie, E. (ed.), Nationalism... a.a.0., S.338-352.

13 J.S. Fumival hat diese Herrschafts- und Wirtschaftsgebilde folgendermaßen umschrieben: "In Burma, as in Java, probably the first thing that strikes the visitor is the medley of peoples Europeans, Chinese, Indians and natives. It is in the strictest sense a medley, for they mix but do not combine. Each group holds by its own religion, its own culture and language, its own ideas and ways. As individuals they meet, but only in the market place, in buying and selling. There is a plural society, with different sections of the community living side by side, but separately, within the same political unit. Even in the economic sphere there is a division of labour along racial lines. Natives, Chinese, Indians and Europeans all have different functions, and within each major group subsections have particular occupations...", Furnival, J.S., Colonial Policy... a.a.0., S.304-305. 
Der nachkoloniale Territorialstaat kann und will an dieser ethnisch orientierten Ordnung der Wirtschaft und Herrschaft nicht mehr festhalten. In seinem Selbstverständnis und nach dem Wortlaut seiner Verfassung ruht er nicht mehr auf einzigartigen und unvergleichbaren ethnischen Kollektiven, sondern er steht jeweils verschiedenen, aber gleichberechtigten, verrechenbaren und zur Selbstbestimmung befähigten Individuen gegenüber. In seiner Verfassung hat er sich zumeist verpflichtet, diese Individuen nicht aufgrund jener Merkmale diskriminieren zu wollen, auf deren Grundlage ethnische Gruppen sich oft definieren Sprache, Religion, Rasse, Herkunft.14 Er kann ebensowenig die Individuen auf der Grundlage dieser Merkmale politisch oder wirtschaftlich begünstigen. Damit führen die neuen Rechtsansprüche - der Chancengleichheit, der Freizügigkeit, der Berufsfreiheit - gegenüber überkommenen ethnisch geprägten Wirtschafts- und Herrschaftsordnungen zu neuen Chancen und Drohungen. Die Durchsetzung des Territorialstaates und wirtschaftliche und politische Modemisierung begründen neue Strukturen: Einheitliche, "nationale" und zentralisierte Arbeits-, Rohstoff- und Produktenmärkte entstehen, zentralisierte Verwaltungs- und Parteiorganisationen beginnen sich über die gesamte Staatsfläche zu erstrecken.

Spezialisierte und regional konsolidierte ethnische Gruppen versuchen in andere Wirtschaftszweige und in ferne oder nationale Märkte einzudringen und fürchten zugleich, zum Opfer des gegenläufigen Versuchs anderer ethnischer Gruppen zu werden. Die eigene ökonomische Differenzierung und Expansion wird unter den Rechtsansprüchen der Berufsfreiheit, Freizügigkeit und Chancengleichheit vorangetrieben, die gegenläufige Differenzierung und Expansion der anderen wird als Bedrohung einer ethnischen Identität, eines ethnischen Berufsideals oder Siedlungsgebiets verurteilt. Die Durchsetzung einer Zentralmacht, ihrer Verwaltungs- und ihrer Parteiorganisationen setzt die gleichen Ambitionen und Ängste frei. 15

Der wachsende Wettbewerb um neue Wirtschaftsbereiche und Märkte führt aber nicht nur zu einem Kreislauf "ethnischer" Kritik, der neue Wettbewerbszwang führt zu einer verstärkten Solidarität und manchmal zu einer Erweiterung der ethnischen Gruppen. Händler, Unternehmer, Handwerker und Arbeiter einer ethnischen Gruppe beginnen sich zu organisieren, um "ihre" Wirtschaftszweige und Märkte besser verteidigen, um in fremde besser eindringen zu können. Der Prozeß einer Rekonstruktion ethnischer Identität macht es möglich, mit Hilfe des Arguments der Sprachverwandtschaft, der gemeinsamen Geschichte, der Nachbarschaft oder der Abstammungs- und Rassegemeinschaft, weitere Gruppen der eigenen anzuschließen. Eine erneuerte und reflektierte ethnische Identität dient damit neuen

14 Rothermund, D., Ethnische Konflike, in: Nohlen, D., Waldmann, P. (eds.), Pipers Wörterbuch zur Politik, Bd. 6., München 1983, S.178-186.

15 Horowitz, DL., Ethnic Groups in Conflict, Berkeley 1985, "Economic Interests", S. 105-135. Siehe auch: "Ethnicity in the words of a cynic entails not the collective will to exist but the existing will to collect.", S.104. 
Wettbewerbsinteressen, sie richtet sich gegen andere ethnische Gruppen und sie erscheint einer wachsenden Zahl von Gruppenmitgliedern sinnfällig und nützlich. Ethnische Kritik und ethnische Organisationen eines gesteigerten Wettbewerbs bilden aber nur ein Element eines umfassenderen Beurteilungsmusters und nur eine erste Etappe eines umfassenderen Verteilungskampfes.

\section{Die neuen Erwartungen}

Der neue Wettbewerb verändert die ethnisch geprägte Wirtschafts- und Herrschaftsordnung, aber er überwindet sie nicht. Das neue Wettbewerbsmuster weckt aber zugleich das Bedürfnis ethnischer Gruppen - jenseits ihres Anspruchs auf Einzigartigkeit -, die politische, ökonomische und kulturelle Stellung ihrer Gruppe einem umfassenden Vergleich zu unterziehen. Indem sich nach der Unabhängigkeit die Durchsetzung des Territorialstaats vollendet, einheitliche und zentrale Partei- und Verwaltungsapparate durchsetzen, einheitliche und zentrale Märkte entstehen, eine einheitliche, eine nationale Kultur und einheitliche, nationale Informationsmedien begründet werden, entsteht zugleich ein neuer Rahmen und ein Maßstab, auf dessen Grundlage sich der "nationale" politische, ökonomische und kulturelle Stellenwert der einzelnen ethnischen Gruppe bestimmen läßt. Die personelle Zusammensetzung der Verwaltungs- und Parteiorganisationen, Wirtschaftsstatistiken, die Inhalte der Schulbücher, Radio- und Fernsehsendungen können Auskunft über die Macht, das Einkommen und das Prestige ethnischer Gruppen geben. 16

Der wachsende ethnische Wettbewerb setzt bei ethnischen Gruppen das Bedürfnis nach einem Vergleich, einer Bestimmung ihres Stellenwerts frei, durch Durchsetzung des Territorialstaats und die beschleunigte Modernisierung machen einen Vergleich möglich. Die Gruppen mögen einander fremd und unverzichtbar bleiben, aber sie sind nicht mehr unvergleichbar. Der zentralisierende und modemisierende Territorialstaat begründet aber nicht nur die Chance eine umfassenden Vergleichs, er begründet zugleich neue Erwartungen.

Er begründet die Erwartung auf mehr Wohlstand, auf soziale und geographische Mobilität und er begründet die Hoffnung auf wachsende soziale Gleichheit. Diese soziale Gleichheit soll an die Stelle traditionell akzeptierter oder einer erst gar nicht wahrgenommenen sozialen Ungleichheit treten. Diese soziale Gleichheit bleibt auf ein neues, ein "meritokratisches" Ideal verpflichtet. 17 Sie zeigt sich in der Ungleichheit individuellen Verdienstes, erwachsen aus einer Gleichheit der - politischen, ökonomischen und sozialen - Chancen. Der Anspruch auf Chancengleichheit führt zugleich zu der Annahme, daß individueller Verdienst gleichmäßig über alle ethnischen Gruppen verteilt sein müsse. Diese Annahme der Gleichvertei-

16 Baechler, J., Les Phenomenes Revolutionnaires, Paris 1970, S.38-39.

17 "Ability x Effort = Merit"., Young, M., The Rise of the Meritocracy, London 1958. 
lung von individuellem Verdienst - in der Form von Macht, Einkommen oder Prestige über alle ethnischen Gruppen begründet einen Maßstab zur Beurteilung der traditionellen Verteilungsmuster.18 Diese Beurteilung führt aber stets zu einem Kreislauf ethnischer Kritik an den Verteilungsmechanismen der Macht, des Einkommens und des Prestiges.

Es ist äußerst unwahrscheinlich, daß die Verteilung von Macht, Einkommen und Prestige im Rahmen einer idealen Verteilungsgerechtigkeit oder einer idelalen Verteilungsungerechtigkeit erfolgt. Vormoderne, ethnisch heterogene Gesellschaften sind weder vollendet egalitäre Konföderationen noch vollendet hierarchisierte Kastengesellschaften. Es ist zugleich äußerst unwahrscheinlich, daß sich diese relativen und vieldeutigen ethnischen Verteilungsungerechtigkeiten genau werden bestimmen lassen. Ethnische Gruppen werden sich selten auf einen einheitlichen Maßstab der Beurteilung ethnischer Verteilungsgerechtigkeit einigen können. Der Verleich ihres politischen, wirtschaftlichen und kulturellen Stellenwerts wird einer ethnischen Gruppe damit oft die eindeutige Beobachtung und immer den einseitigen Verdacht einer ethnischen Benachteiligung gestatten. Diese Beobachtungen und Verdächte machen eine erste umfassende ethnische Kritik an den Verteilungsmechanismen von Macht, Einkommen und Prestige möglich.

Diese Kritik fügt zahlreiche Einzelbeobachtungen zusammen und vereinigt sie zu einer Gesamtdarstellung der ethnischen Gruppe. Diese Gesamtdarstellung bestimmt zugleich den Stellenwert der Gruppe gegenüber anderen und im Rahmen eines Staates. Die Kritik leistet damit einer neuen, einheitlichen Konzeption der Gruppe und einer neuen Orientierung auf ein Zentrum, den Staat und seine Verteilungsmechanismen, Vorschub. Der modemisierende Staat begründet aber nicht nur neue Erwartungen und Urteilsmaßstäbe, er setzt - wie wir bereits gesehen haben - Entwicklungen frei, die ethnische Verteilungsmuster verändern, aber nicht überwinden. Damit wird ein weiterer Kreislauf umfassender ethnischer Kritik freigesetzt. Verringert sich beispielsweise die sozi-ökonomische Distanz zwischen einer als "überlegen" und einer als "unterlegen" eingestuften ethnischen Gruppe, so kann diese Verringerung von der ersten Gruppe als Bedrohung, von der zweiten als ungenügend interpretiert werden.19 Der modemisierende Staat begründet damit die Möglichkeit, das Bedürfnis und den Beurteilungsstandpunkt eine umfassenden "ethnischen" Vergleichs. Er macht damit ethnische Verteilungsmuster offensichtlich und zugleich illegitim. Indem er

18 "If the $20 \%$ of the population living below the poverty level are all in one ethnic group, an issue of discrimination and social policy is posed. If the poor represent each of the country's ethnic groups in proportion to their population, it is an issue of economic policy. Thus a rearrangement of ethnic groups among the poor does not in itself reduce the misery within a population.", Weiner, M., The Pursuit of Ethnic Equlity through Preferential Policy: A Comparative Public Policy Perspective, in: Goldmann, RB./Jeyaratnam Wilson, A. (eds.), From Independence to Statehood, Managing Ethnic Conflict in 5 African and Asian States, New York 1984, S.63-81, S.66.

19 Im Rahmen der Revolutionstheorien hat T.R. Gurr auf dieses Phänomen der relativen Deprivation aufmerksam gemacht. Gurr, TR., Why Men Rebel, Princeton 1970. 
sie verändert, aber nicht überwindet, wird er von einem Kreislauf wechselseitiger ethnischer Kritik und einer wachsenden ethnischen Organisation der Verteilungskämpfe begleitet.

Bislang unverbundene Bereiche der kulturellen Einschätzung, der ökonomischen Integration, der politischen und administrativen Repräsentation können nun zusammengefaßt und einheitlich beurteilt werden. Vereinzelte Konflikte oder individuelle Klagen und Ressentiments können nun in einem wachsenden Dossier ethnischer Benachteiligung Eingang und Erklärung finden. Im Gegenzug können in diese Sachgebiete und Einzelkonflikte ethnische Forderungen eingebracht werden. Dies setzt einen weiteren Kreislauf der ethnischen Organisation, der Begründung von Kultur- oder Wirtschaftsorganisationen frei. Indem diese Organisationen in die jeweiligen kulturellen, sozialen und ökonomischen Konflikte hineingezogen werden, werden die Standpunkte der Konfliktbeteiligten prinzipieller und intransingent, die Konflikte intensiver. Diese Entwicklung droht die ethnischen Gruppen in wachsendem Maße zu einer Partei zu konsolidieren und verstärkt das Bedürfnis, die Konflikte in eine politische Sphäre zu verlängern und zu übertragen. Die Politisierung ethnischer Gruppen und ethnischer Konflikte ist aber nicht selbstverständlich und bedarf der gesonderten Betrachtung.

Zuvor sollen aber die bislang aufgezeigten Wirkungen des Modemisierungsprozesses resümiert werden. Die Durchsetzung des Territorialstaats und die Beschleunigung des Modemisierungsprozesses verändern, aber zerstören nicht die ethnischen Identitätsmuster. Stattdessen zeigen diese Muster eine unvorhergesehene Lebendigkeit, eine Anpassungsfähigkeit und Nützlichkeit. Eine "Modemität der ethnischen Traditionen" wird sichtbar, bei der sich ethnische Tradition und Moderne in einer Weise verbinden, daß der Tradition ihr Geltungsanspruch, aber nicht unbedingt ihr Inhalt verbleibt. 20 Der Wert ethnischer Identität, ethnischer Tradition liegt stattdessen gerade in dem Anschein ihrer Kontinuität. Dieser Schein der Kontinuität verdeckt, daß der vermeintliche ethnische Traditionsschatz oft das Resultat einer mit selektiven, wissenschaftlichen Mitteln betriebenen Neuschöpfung ist. Er verdeckt, daß ethnische Gruppen sich zum Zweck des sozialen Aufstiegs, des Wettbewerbs und der Expansion auf der Grundlage dieser Identität solidarisieren, reorganisieren und erweitem. Der Schein der Kontinuität verdeckt auch, daß sich die ethnische Gruppenorganisation von einem festen Ordnungsmuster traditioneller Wirtschafts- und Herrschaftsformen nun zu einẹr Gruppenstrategie im sozio-ökonomischen Verteilungskampf verwandelt.

Die Wirkungen des Modemisierungsprozesses erscheinen deshalb widersprüchlich, fast ironisch. Seine neuen Identitätsmuster lösen eine Idealisierung der alten, ethnischen aus. Seine neuen, großräumigen Markt-, Wirtschafts- und Verwaltungsstrukturen erzwingen

20 Lloyd I. Rudolph und S. Hoeber Rudolph haben dieses Entwicklungsmuster am Beispiel Indiens umfassend dargestellt: The Modemity of Tradition, Political Development in India, Chicago 1967. 
eine Reorganisation und gestatten das Eindringen ethnischer Gruppen. Seine neuen Erwartungen gesamtgesellschaftlicher Entwicklung und wachsender Verteilungsgerechtigkeit lösen eine umfassende ethnische Beurteilung und Organisation des Verteilungskampfes aus.

Die Idealisierung ethnischer Identität, die Reorganisation ethnischer Gruppen und dieser umfassende ethnische Beurteilungs- und Interessenstandpunkt intensivieren oder begründen zugleich kulturelle, ökonomische und soziale Konflikte. Eine idealisierte Gruppenkultur will nicht nur geduldet, sondern bald auch respektiert werden. Neben den Stolz auf die eigene, tritt rasch die Abwertung der fremden Gruppenkultur. Die ethnische Reorganisation des Wettbewerbs zielt auf die ökonomische Abwehr oder die Verdrängung des ethnischen Gegners. Die ethnische Beurteilung der Verteilung von Macht, Einkommen und Prestige führt zum ethnisch organisierten Verteilungskampf, zur Verteidigung der ethnischen "Besitzstandsinteressen".

Aber diese Konflikte müssen weder von schrankenloser Militanz sein, noch sind es spezifisch politische Konflikte. Es handelt sich noch nicht um Konflikte, die sich direkt auf ein politisches Zentrum, die Instanz des Staates - seine Verwaltung, Rechtssprechung, Exekutive und Legislative - ausrichten. Die ethnischen Gruppen sind noch nicht in der Parteienstruktur verankert und die Konflikte haben sich noch nicht zu einem parlamentarischen oder grenzenlosen Kampf um die politische Macht verwandelt. Es sind aber diese politischen, also die politisierten ethnischen Konflikte, die die Neutralität des Staates in Frage stellen können, seine demokratischen Grundlagen denaturieren, entweder seine Gestalt verändern oder seine Einheit gefährden. Diesen Prozeß der Politisierung und seine Konsequenzen müssen wir nun betrachten.

\section{Die Politisierung ethnischer Konflikte}

\section{A. Die Allmacht des modernisierenden Staates}

Die Politisierung ethnischer Konflikte wird möglich und erleichtert durch die neue Machtfülle des modernen Territorialstaates und durch seine demokratischen Grundlagen. Gegenüber allen ihm vorangegangenen politischen Regimen verfügt der modeme Territorialstaat über eine historisch neuwertige, bürokratische Kontrollgewalt. Er verfügt nicht nur über eine neue Machtfülle, er beansprucht sie auf der Grundlage einer neuen, demokratischen Legitimität und sie wird ihm zugeschrieben. In dem Maße, in dem er, im Rahmen des Kolonialisierungsprozesses, erst seit kurzem an die Stelle vorgängiger, traditioneller Herrschaftsformen getreten ist, erscheint diese Machtfülle noch beeindruckender. Sie steigert sich in dem $\mathrm{Maße}$, in dem der Staat zum Träger eines beschleunigten und nachgeschalteten Modemisierungsprozesses wird. Indem der Staat sich freiwillig oder unfreiwillig unter die 
Imperative einer beschleunigten sozialen und wirtschaftlichen "Entwicklung" gerückt sieht, wird ihm eine Vielzahl traditioneller Strukturen zugänglich, erscheinen sie ihm als disponierbar und veränderungsbedürftig. Das Ideal und der Anspruch auf nationale Entwicklung weckt die hegemonialen Instinkte dieser neuen Herrschaft.

Diese wachsende, reale, beanspruchte und zugeschriebene Machtfülle erleichtert es ethnischen Gruppen, ihre jeweils bilateralen, wirtschaftlichen, sozialen oder kulturellen Konflikte vor die Instanz eines Staates zu tragen, der, nachdem er auch in entlegene soziale Sphären eindringt, sich nun auch mit dem Anspruch konfrontiert sieht, in all diesen Sphären lokale oder ihm sachfremde Konflikte schlichten zu müssen.21

Diese Einschätzung des Staates als einer für alle Konfliktlösungen zuständigen und formal ethnisch neutralen Instanz wird aber durch die wachsende Radikalisierung ethnischer Konflikte rasch korrigiert. An die Stelle der Annahme seiner ethnischen Neutralität tritt der begründete oder unbegründete Verdacht seiner ethnischen Parteilichkeit und das Interesse, ihn für die Zwecke der eigenen Gruppe zu nutzen. Mangelnder Zugang zur Staatsmacht gilt nun als notwendiger Vorteil, der an die gegnerische Gruppe verspielt wurde. Der Staat gilt als eine Waffe, die sich solange gegen die eigene Gruppe richten wird, wie man sie nicht gegen die fremde richtet. Das heißt, der Staat wird nun im Lichte zweier Modelle, des Nullsummenspiels und des präventiven Erstschlags interpretierbar. Diese neue "ethnische" Einschätzung des Staates löst dann rasch die nur scheinbar widersprüchliche Konsequenz aus, daß gerade jene Gruppen, die sich ein hohes $\mathrm{Ma} \beta$ an Distanz zum Staat erhoffen, die weder Integration noch Akkulturation wünschen, in einen erbitterten Wettbewerb um die Sicherung staatlicher Macht getrieben werden. Je kleiner und ohnmächtiger die ethnischen Gruppen desto stärker müssen sie die Situation fürchten, in der die Staatsmacht in die Hände größerer und überlegener Gruppen gerät. Ihre Versuche, sich staatliche Macht zu sichern, ihre politischen Forderungen drohen deshalb rasch über das Maß dessen herauszugehen, was berechtigt oder durchsetzbar erscheint. Ein fataler Erfahrungsprozeß bricht sich Bahn, bei dem kleine ethnische Gruppen der Intransigenz, große Gruppen ethnischer Hegemonialinteressen bezichtigt werden.

Bürokratische Verwaltung und die Ambition eines beschleunigten Entwicklungsprozesses steigern erst die Macht des Staates und dehnen schließlich ihre Grenzen aus.

Eine Grenzsituation wird denkbar, in der eine bürokratisierte Staatsmacht in vielerlei soziale, kulturelle und wirtschaftliche Teilbereiche einzudringen beginnt, mit dem Rechtsanspruch ihrer Entwicklung, ihrer Nationalisierung und Modernisierung. Die Entstehung und die Unabhängigkeit der Staaten der Dritten Welt ist aber in vielen Fällen inner-

21 Rothschild, J., Ethnopolitics... a.a.0., S.4-5, "Both dominant and subordinate groups come to view the state as the gatekeeper of the contradictions and the controller of the conflict." 
halb einer so kurzen Zeitspanne erfolgt, daß sich eine eigenständige, gruppenunabhängige Tradition des Nationalismus - eigenständige Erfahrungen, Symbole, Rituale, Historiographien einer nationalen Identität - nicht entwickelt hat. Sofern ethnische Konflikte nicht bereits während des Unabhängigkeitskampfes ausgebrochen sind, drohen sie wenig später auszubrechen. Entweder schon vor oder nach der Unabhängigkeit erweist sich aber die Abwehr, die scheinbare Prävention solcher Konflikte, der Prozeß des "nation building" oft als kontraproduktiv. In Ermangelung eigenständiger, rezenter "nationaler" Erfahrungen und Symbole neigt eine dominante ethnische Minderheit oder Mehrheit rasch dazu, ihre ethnische Identität mit einer nationalen zu verwechseln. In die neue Begriffsform der Nation kippt sie den Inhalt ihrer spezifischen ethnischen Identität. Der vorgebliche Prozeß nationaler Homogenisierung verwandelt sich damit zum Mittel ethnischer, kultureller Gleichschaltung und beschleunigt die Konsequenz ethnischer Segmentalisierung. Der Staat dehnt nicht nur seine Grenzen aus, unter dem Mantel nationaler oder nationalisierender Entwicklungspolitik betreibt er evtl. den Prozeß der ethnischen Homogenisierung von Staat und Gesamtgesellschaft. Damit radikalisiert er endgültig jene Widerstände und Gegenspieler, die er ursprünglich vorgab, integrieren zu wollen.22

Der Territiorialstaat ruht aber auf demokratischer Grundlage. Auf die Instanz des Staates ausgerichtet, von seinen Maßnahmen bedroht, beginnen ethnische Gruppen seine demokratischen Grundlagen, seine Parteienstruktur, seine Legislative für ihre Zwecke zu nutzen.

\section{B. Die Zerstörung der demokratischen Grundlagen}

Ich will im folgenden zuerst die Form und die Wirkung einer solchen ethnischen Parteipolitik betrachten und daran anschließend nach den Motiven fragen, die Wählem die

22 Hugh Seton-Watson urteilt über den Wert und die Wirkung dieser nationalistischen Doktrinen wie folgt: "In the case of many, though not of all, nations there has been a further task for nationalists: to build a nation within an independent state, by extending down to the population as a whole the belief in the existence of the nation, which before independence was won, was only held by a minority... (This nationalist doctrine) has inspired immense outputs of thetoric, and each brand has its own peculiarities, some of which must be admitted to be picturesque, though literary distinction and beauty are qualities which I should hesitate to attribute to them... Nationalist doctrines will no more stand up to critical analysis then any other ideologies... Nationalism has been responsible for floods or metoric and for the debasement of human language. Nationalists have shown ignorant contempt for institutions, customs and beliefs, which had proved their worth for centuries and have replaced them with fragile structures and empty slogans. Extreme nationalism has been a crude substitute religion, replacing withered faith by fanatical hatreds. Too often its leaders have been frustrated social misfits and self-important semi-intellectuals. At its worth, extreme nationalism has led to massacres and forcible expulsions of millions of mainly innocent people..." H. SetonWatson, Nations and States, an Inquiry into the Origins of Nations and the Politics of Nationalism, London 1977, S.3,12. 
Bevorzugung einer ethnischen Partei, Politikem die Begründung einer ethnischen Partei erleichtern. Diese Reihenfolge wurde gewählt, um zwei verbreiteten aber irreführenden Einschätzungen ethnischer Politik entgegenzutreten. Die Einschätzung, ethnisch-motivierte Parteipolitik sei nach den bisherigen Ausführungen ein verständliches, fast zwangsläufiges Resultat und ethnische Parteien seien im Rahmen und an der Seite traditioneller liberaler und sozilistischer Parteien zwanglos zu akkomodieren. Ich will stattdessen darauf hinweisen, daß trotz der verbreiteten Existenz ethnischer Konflikte, deren Verankerung in einem Parteienspektrum keineswegs leicht und selbstverständlich ist. Wenn allerdings diese Schwelle überschritten ist, so gehen von einer ethnischen Parteipolitik Wirkungen aus, die eine Demokratie zu denaturieren drohen. Ich betrachte mithin erst die Immunität, schließlich die spezifische Verletzlichkeit der demokratischen Herrschaft gegenüber Formen der ethnischen Willensbildung und der ethnischen Parteipolitik, die ihr inkompatibel sein müssen.

Ethnische Parteien lassen sich provisorisch umschreiben als Parteien, die einen Ausschnitt der gesamten Wählerschaft, also eine bestimmte ethnische Gruppe, ansprechen. Sie wecken exklusive, ethnische Interessen oder sie entsprechen ihnen. Sie sprechen Wähler unter ihrer ethnischen Identität an. Eine solche Strategie muß unter dem machtpolitischen Interesse der unbegrenzten Stimmenmaximierung, ebenso wie unter dem gesamtgesellschaftlichen Interesse umfassender Themen- und Problemrelevanz als unvernünftig erscheinen. Vor allem im Falle ethnischer Minderheiten verzichtet die Partei auf eine Strategie des maximalen Stimmen- und damit des unmittelbaren Machterwerbs. Alle ethnischen Parteien verzichten darauf, zu gesamtgesellschaftlichen Problemen Stellung zu nehmen und diese lösen zu wollen. Die Begründung einer etnischen Partei stellt sich deshalb keineswegs als die problemlose Verlängerung eines bereits bestehenden ethnischen Konfliktes in die politische Sphäre dar. Zumindest kleinere Gruppen nehmen mit dieser Entscheidung oft Abschied von der Hoffnung auf den Zugang zur Macht und handeln sich zugleich den Vorwurf einer Aufkündigung eines verbindlichen demokratischen Konsenses ein. Da ethnische Parteien zumeist im Rahmen eines etablierten, liberalen, sozialistischen oder nationalen Programmen verpflichteten Parteienspektrums entstehen, sehen sie sich nicht nur der Verachtung, sondem auch dem Druck einer politischen Elite ausgesetzt.23

Ist aber eine ethnische Partei entstanden, so gehen von ihrer Existenz weitreichende Wirkungen aus. Auch in einem Staatswesen, in dem mehrere beispielsweise vier größere ethnische Gruppen existieren, engt bereitsdie Existenz lediglich einer erfolgreichen ethnischen Partei das Stimmenpotential der daneben bestehenden liberalen, nationalen oder sozialistischen Parteien ein. Der Erfolg ethnischer Politik setzt sie bereits dem Verdacht oder dem Anspruch aus, unter dem Vorwand sozialistischer, nationaler oder liberaler Forderungen Interessen der anderen ethnischen Gruppen zu vertreten. Das erfolgreiche 
Auftreten einer ethnischen Partei erleichtert damit die oft unausgesprochene Orientierung bestehender Parteien auf eine jeweils andere ethnische Klientel. In dem Maße, in dem sich eine solche ethnische Orientierung Bahn zu brechen beginnt, verändert sich aber bald die Form des Wahlkampfs und des Wahlaktes. Von einer privaten, individuellen Entscheidung verwandelt sich der Wahlakt zu einer öffentlichen Demonstration ethnischer Solidarität. Die Parteiführer halten Heerschau, sie dokumentieren die numerische Stärke ihrer ethnischen Gruppen. Der Wahlkampf verliert die Züge einer gesamtgesellchaftlichen Auseinandersetzung, die eine Vielzahl von Wählern mit einer Vielfalt von Problemen in einem Wettstreit der Problemlösungen, Versprechungen und Ideen konfrontiert. Der Wahlkampf findet vor getrennten ethnischen Auditorien statt. Siedeln die ethnischen Gruppen räumlich getrennt, so verzichten die Parteien bald darauf, in fremdethnischen Wahlkreisen Kandidaten zu nominieren. Tun sie es dennoch, so bleibt trotz dieser ethnischen Kosmetik die Bindung der jeweiligen Partei an eine ethnische Gruppe für die anderen offensichtlich. Sind die Merkmale der ethnischen Gruppe leicht und öffentlich bestimmbar, so homogenisieren sich die jeweiligen Zuhörer der Wahlveranstaltungen.24 Der jeweils Fremde will nicht nur, man läßt ihn auch nicht zuhören. In dem Maße, in dem sich die Ethnisierung der Parteipolitik vollendet, werden die Wahlergebnisse vorhersagbar, zu einem Abbild der numerischen Stärke der ethnischen Gruppen. Nach einer Phase der hohen Wahlbeteiligungen kann sich oft wachsende Apathie und Stimmenthaltung auf seiten ethnischer Minderheiten zeigen. Der demokratische Prozeß beginnt zumindest bei ihnen an Glaubwürdigkeit zu verlieren.

Der Prozeß der Ethnisierung der Parteien wiederholt nicht nur die Segmentalisierung der Gesamtgesellschaft, er wird schließlich zu einem wesentlichen Antrieb dieser Segmentalisierung. Die ethnischen Verteilungs- und Konkurrenzkämpfe, die ethnischen Ansprüche auf kulturelle Anerkennung und Respekt werden politisch und radikal. Der demokratische Mechanismus, idealerweise ein Mechanismus der Kompatibilisierung vielfältiger und gegenläufiger Interessen, operiert in wachsendem Maße gerade in der entgegengesetzten Richtung einer beständigen Erweiterung der ethnischen Distanz und der ethnischen Ressentiments. Die Segmentalisierung verstärkt sich, wenn sich mehrere ethnische Parteien um die Gunst der Wähler einer ethnischen Gruppe bemühen. Dieser politische Wettbewerb im Binnenraum einer ethnischen Gruppe radikalisiert auf Dauer die jeweiligen Forderungen

24 D. L. Horowitz nennt einige Beispiele: "A member of a Commonwealth team sent to observe the 1964 Guyanese election, described it as a 'racial census' in which a person's vote was predetermined by the demands of his (security) 'in an atmosphere charged with fear"'. Auf Trinidad, wo ein kreolischer Wählerblock einem indischen entgegensteht, wurde von den Kreolen während des Wahlkampfs der folgende Kalypso gesungen: "We don't want no coolie premier, we don't want no roti (indisches Brot) govemment." Auf Trinidad kann ein Kabinettsminister, der - indischen P.N.M. Partei während einer Wahlveranstaltung sagen: "I do not see any DLP faces around." Er meint die Kreolen der Democratic Labour Party., Horowitz, DL., Ethnic Groups... a.a.0., S.322, $326,346$. 
einer ethnischen Besitzstandssicherung oder Expansion und treibt die Gruppen und ihre Parteien immer weiter auseinander. Dieser Kreislauf der Radikalisierung bedroht in immer stärkerem Maße die noch verbliebene politische Einheitlichkeit der Gesamtgesellschaft.

Schließlich bildet lediglich das Zentrum der Macht - Regierung, Verwaltungsapparat und Militär - diejenige Klammer, die eine in ethnische Enklaven zerfallende Gesamtgesellschaft noch zusammenhält. Aber auch dieser Zusammenhalt wird prekär, wenn die Größenverhälmisse ethnischer Gruppen einer einzelnen Gruppe keine politische Mehrheitsfähigkeit gestatten, sichern multiethnische Koalitionen und Allianzen den Zusammenhalt. Dieser Zusammenhalt ist aber prinzipiell instabil, weil er in letzter Instanz auf nicht verallgemeinerungsfähigen Interessen, auf inkompatiblen Identitäten beruht. Eine wachsende Instabilität kann sich damit im Zentrum des Staates Bahn brechen. Diese Instabilität kann schließlich die einzig verbliebene vorgeblich ethnisch neutrale Institution, das Militär, in die Bahnen ethnischen Streits ziehen und zur Intervention treiben.

Wenn eine ethnische Mehrheitsgruppe gegen eine oder mehrere Minderheiten steht, so kann sie die Minderheiten oft über Jahre hinaus vollständig von der Regierungsbeteiligung, vom Verwaltungsapparat und Militär ausschließen. Sie kann ein Programm der Wirtschaftsentwicklung und des "nation-building" zum Zwecke der ausschließlichen Durchsetzung dieser Mehrheitsinteressen vorantreiben. Sie kann die Minderheiten gegeneinander ausspielen und spalten.

Die Minderheitenforderungen nach Dezentralisierung, am Ende nach einem Separatstaat, dienen schließlich nicht mehr einem taktischen Kalkül der Sicherung kleinerer Konzessionen, sie wandeln sich zu einem Ideal, einem eigenständigen Programm, am Ende zu einem Kampf, der sich selbst der Kontrolle der Minderheitenparteien entziehen kann. Die Drohung oder die Entwicklung eines Bürgerkriegs verleiht auch in diesem Falle dem Militär eine wachsende Bedeutung. Das Militär soll den Politikem zu einer endgültigen militärischen Lösung verhelfen, oder es wird seinerseits intervenieren, versuchen im Rahmen einer Militärherrschaft eine politische Lösung herbeizuführen.25

Angesichts erst der Schwierigkeit, schließlich der Zerstörungskraft ethnischer Politik stellt sich die Frage, welchen Motiven auf Seiten der Wähler ebenso wie der Parteipolitiker eine solche Politik entspricht. Welche Motive treiben beide Gruppen dazu, eine solche machtpolitisch und oft gesamtgesellschaftlich immer unvernünftige Strategie zu verfolgen?

In dem Maße, in dem der demokratisch verfaßte Territorialstaat, mithin seine Parteien, sich zu politischen Trägern eines nachgeschalteten und beschleunigten Modemisierungsprozes-

25 Horowitz, DL., Coup Theories and Officers' Motives: Sri Lanka in Comparative Perspective, Princeton 1980. 
ses machen, drohen die nicht erfüllten Erwartungen eines solchen Modemisierungsprozesses die Glaubwürdigkeit eben jener Parteien und Programme in Zweifel zu ziehen. Liberale und sozialistische Parteien sind in besonderem Maße einem solchen Ideal gesamtgesellchaftlicher Entwicklung verpflichtet. Im Maßstabe enttäuschter Erwartungen droht dieses Ideal nationaler Entwicklung durch das Problem der sozio-ökonomischen Verteilungskämpfe ersetzt zu werden. Diese Verteilungskämpfe können aber in noch weitgehend traditionalen Gesellschaften leichter nach dem Kriterium der ethnischen Gruppenzugehörigkeit als nach den weitgehend abstrakten der sozio-ökonomischen Lage, der Klasse oder der individuellen, "meritokratischen" Verdienstansprüche geführt werden.

Für die Durchsetzung ethnischer Gruppeninteressen aber werden ethnische Parteien gerade begründet. Sie können direkt, manchmal schamlos, Interessen der ethnischen Besitzstandssicherung oder -erweiterung artikulieren, während liberale und sozialistische Parteien einem nationalen Wachstum und einem ethnisch neutralen Bild des Wählers verpflichtet, sich schwerer tun im Schatten ihrer jeweiligen liberalen oder sozialistischen Entwicklungsalternativen sich eine ethnische Wählerklientel zu sichern. Oft gelingt es aber auch ihnen mit Hilfe einer doppelten, expliziten und impliziten politischen Rhetorik und Symbolik, sich ethnischen Gruppen zu verpflichten.

Der Modernisierungprozeß zum - unerreichbaren - politischen Handlungsideal erhoben, droht damit die mit ihm verbundenen Parteien und Programme zu desavouieren und mit der Wende zu einem neuen Realismus gewinnen schließlich ethnische Parteien eine wachsende Attraktivität. Diese Abkehr von einem nationalen Wachstumsideal löst zugleich die weit schwerwiegendere von einem politischen Entwicklungsprozeß aus. Der Niedergang liberaler oder sozialistischer Parteien begründet eine - nicht nur gegenüber eurozentrischen Maßstäben - regressive politische Entwicklung.

Parteien wecken und entsprechen Interessen ihrer Wähler. Sie verstärken oder begründen sie. Die Parteien sprechen ihre Wähler damit unter einem unmittelbar sinnfälligen Aspekt oder eher abstrakten Konstrukt ihrer Identität an. Diese selektierten oder begründeten, auf Dauer gestärkten Interessen prägen die Wähler, aber auch die jeweiligen Parteien. Eine vom Kriterium des Wahlerfolgs determinierte, vorangetriebene, wechselseitige politische Sozialisation bricht sich Bahn. Die politischen Interessen, die ihnen entsprechenden Identitätsaspekte oder die von ihnen begründeten Identitätskonstrukte erscheinen aber in dem Maße plausiber, in dem die Parteien den Staat und die Gesellschaft diesen Interessen entsprechend verwandeln können. Den Interessenmustern müssen zumindest auf Dauer, wenn nicht von Anfang an gesellschaftliche Strukturen entsprechen, diese als sinnfällig und notwendig erweisen.

Indem liberale oder sozialistische Parteien Wähler beispielsweise unter individualistischen Interessen oder Interessen einer bestimmten sozio-ökonomischen Lage ansprechen, indem 
sie schließlich Staat und Gesellschaft im Sinne dieser Interessen, im Lichte dieser Annahmen umbilden, bringen sie schließlich Strukturen zur Geltung, die diese Interessen im politischen Kontext als sinnfällig oder praktisch erweisen. Parteien lösen damit nicht nur eine politische Erziehung und eine politische Transformation aus, sondem vermitteln beide Prozesse einander. Der Erfolg dieser Parteien wird abhängig von dieser doppelten Leistung. Die gesellschaftliche Umwandlung macht relativ abstrakte, individualistische oder kollektive, sozio-ökonomische Identitätsmuster schließlich plausibel, die politische Sozialisation macht aber diese gesellschaftliche Umwandlung erst denkbar und möglich.

Im Rahmen einer noch weitgehend traditionalen Gesellschaft sprechen liberale und sozialistische Parteien ihre Wähler deshalb unter Identitätsaspekten und konstrukten an, die noch wenig sinnfällig erscheinen. Ihr Bild des Wählers erscheint anachronistisch: Sie sprechen Wähler unter Interessen- und Identitätsfragmenten an, denen erst im Ablauf des Modemisierungsprozesses jene Strukturen entsprechen werden, die diese schließlich als sinnfällig erweisen. Liberale und sozialistische Parteien stellen sich damit auf den Standpunkt einer notwendigen, einer produktiven Illusion: Sie prätendieren das Interessen- und Identitätsresultat des Modemisierungsprozesses in der Hoffnung, ihn dadurch auslösen und vollenden zu können. Mit der Fragwürdigkeit des Modernisierungsprojekts, mit der Entstehung ethnischer Parteien kommt aber ein anderes Interessen- und Identitätsmuster zum Zuge.

Diesen Interessen, diesen Identitätsmustern entsprechen noch weitgehend die bestehenden gesellschaftlichen Strukturen. Das ethnische Identitätsmuster ist vorgegeben, es bildet kein abstraktes Konstrukt, es bildet auch kein Fragment der Identität des Wählers ab, sondern es gibt zu Recht oder zu Unrecht vor, seine gesamte Alltagsgestalt abbilden zu wollen. Ethnische Identitätsmuster sind unmittelbar sinnfällig, umfassend und vorgegeben. Sie unterstellen weder Wandel noch Abstraktion in der Selbstbedeutung. An die Stelle eine politischen Sozialistionsprozesses rückt ein Akt des Wiedererkennens und der Akklamation. Ethnische Parteien gestatten es ihren Wählern, sofern sie sich in dem Entwurf ethnischer Identität wiedererkennen, ihre Alltagsgestalt problemlos in die politische Sphäre zu verlängern. Ethnische Politik, die Artikulation ethnischer Interessen verbinden sich schließlich umstandslos mit der ethnischen Folklore, dem Bekenntnis zur Gruppe, der Prozession und dem Fest.26 Auch unabhängig von dem durch den eventuellen Kollaps der Modemisie-

26 Bipin Chandra Pal hat bereits 1910 in seinem Buch "The Spirit of Indian Nationalism" versucht, der Kongreßbewegung das Programm einer religiösen, ethnischen Partei vorzugeben: "... Statesmanship is not concemed with a rationality, but simply with the reality of popular faith... it is this reality which lends vitality and strength to historic movements... Among the Indus, civil religion is growing through an easy and natural process out of the old symbolism and ritualism of the people. Hinduism has indeed, like all ethnic systems, this advantage over credal religions, that it symbols and rituals... are all partly social and partly spiritual... Consequently the social and the spiritual has found apt vehicles for expressing itself in the current religious rites and formulas of the people. The common Hindu formula for the sacrificial purification of water... has become... a 
rungshoffnung ausgelösten Niedergang liberaler und sozialistischer Parteien verfügen deshalb ethnische Parteien von Anfang an über einen umstandslos evidenten Interessenund Identitätsentwurf. Der Niedergang liberaler und sozialistischer Parteien bildet deshalb nicht die zwingende Voraussetzung und das zwingende Vorspiel einer Entstehung ethnischer Parteien. Dieser Niedergang bildet allerdings das angemessene, düstere Ruinenfeld, vor dem sich diese neuen, scheinbar so natürlichen und realistischen Parteien und Interessen glänzend abheben können.

Die relative und absolute Attraktivität ethnischer Politik motiviert nicht nur Wähler. ethnische Parteien zu wählen, sondern selbstverständlich auch Politiker, ethnische Parteien zu begründen oder bereits bestehende ethnisch zu orientieren.

Im Falle eines noch während der Dokolonialisierung entstandenen, befreiungsrhetorisch wie befreiungsstrategisch überethnischen und westlich orientierten Parteiapparats oder Parteienrepertoirs bildet die Begründung einer ethnischen Partei oft die einzige Möglichkeit, sich eine neue spezifische Wählerschicht zu erschließen. Dies gilt gleichermaßen für Dissidenten innerhalb bestehender Parteien, wie für die Vertreter einer neuen Intelligentsia, die keinen Zugang zu den etablierten Parteien und Führem findet.

Indem sich der demokratische Prozeß zu popularisieren beginnt, indem eine Massendemokratie an die Stelle einer von Honoratioren verwalteten Demokratie tritt, wächst die Attraktivität einer ethnischen Parteigründung. Die Unabhängigkeit und die Erweiterung der politischen Teilnahme läßt nun neue Fragen und Probleme entstehen: Welche Sprache und Religion, welche Kulturen und Regionen sollen von einer neuen nationalen - kulturellen, sozialen und wirtschaftlichen - Entwicklung besonders berücksichtigt werden? In dem $\mathrm{Maße}$, in dem sich eine neue nationale Kultur weder aus dem Nichts noch paritätisch erschaffen läßt, werden eindeutige oder unterstellte Diskniminierungen unausweichlich, die schließlich die Begründung einer ethnischen Partei erleichtem. Die Erweiterung der politischen Partizipation wird darüberhinaus geme theoretisch gefeiert, sie gilt als der überzeugende Nachweis, daß es sich bei diesen Demokratien nicht um das direkte oder indirekte "oktroi" einer europäischen politischen Kultur handele, sondern um ein vollständig assimiliertes, schließlich eigenständiges, politisches Spiel.

Die Entwicklung zur Massendemokratie läßt aber in diesen Ländem keine Masse, sondern ethnische Gruppen in die politische Sphäre treten, die für ihre jeweilige Sprache, Religion, Kultur, und Region, Anerkennung, Toleranz und Unterstützung fordem. Der Prozeß der

symbol of Hindu unity... (the interpretation of the old imagers of gods... has imparted an new meaning... this wonderful transfiguration of the old gods and godesses is carrying the message of new nationalism to the women and the masses of the country...", Kedourie, E. (ed.), Nationalism in Asia... a.a.0., S.345-346. 
Popularisierung zerbricht deshalb, segmentalisiert das bislang diffuse, abstrakte Konzept der nationalen Masse in konkrete und politisch artikulationsfähige Gruppen. Der Prozeß veraltet damit zugleich eine liberalen und sozialistischen Ideen verpflichtete anglo- oder frankophone, laizistische und westlich-orientierte politische Elite.

Durch mangelnde Macht, Alter oder Status von dieser Elite ausgeschlossene Politiker können, sofern sie diesen Prozeß nicht sogar selbst vorantreiben, aus ihm politische Chancen schöpfen.27

Ich habe bislang die Attraktivität einer ethnischen Parteigründung relativ bestimmt. Ethnische Parteien entstehen, weil das klassische Repertoire nicht-ethnischer Parteien bereits besetzt ist und weil nicht-ethnische Parteien oder Unabhängigkeitsbewegungen Erwartungen begründen, die sie nicht erfüllen können. Neben dieser relativen Attraktivität des ethnischen Projekts, neben der relativen Anziehungskraft für einen "ethnischen Unternehmer" besteht aber noch, wie mir scheint, eine absolute, eine allen anderen Altemativen überlegene Anziehungskraft der ethnischen Parteigründung. Dieses Motiv und Kalkül habe ich bereits zu Anfang erwähntund ich will damit schließen:

Jenseits eines Ideals der gesamtgesellschaftlichen Orientierung und Verantwortung, kann jeder Politiker auch als ein rational kalkulierender Unternehmer begriffen werden. Im Rahmen demokratischer Politik wird er seine Machtanteile, die Stimmen, über die er verfügt, so einsetzen, daß er ein Maximum an Einfluß und Wirkung für seine Wähler oder sich gewinnen kann. Unter bestimmten Bedingungen kann eine ethnische Parteigründung tatsächlich ein Maximum an Einfluß und Wirkung erzielen. Von der Gründung einer ethnischen Partei geht latent, von ihren politischen Forderungen gehen bereits vernehmbar eine unverhältnismäßig große Bedrohung der Gestalt und schließlich der Einheit des Staates aus. Je kleiner das vorfindliche oder prospektive Wählerreservoir, je unwahrscheinlicher die Chance eines auf Dauer bedeutenden Stimmenzuwachses, desto größer muß die politische Versuchung sein, Zuflucht zu dieser politischen Option zu nehmen. Sie sichert weniger dem Wähler als dem betreffenden Politiker eine unverhältnismäßig hohe Aufwertung und oft hohe Konzessionen.

Für die betreffenden Politiker wirkt sich diese Ökonomie im Einsatz der politischen Mittel auch in Richtung auf ihre Wählerschaft aus. Indem sie ihre Wähler auf eine Alltagsidentität

27 Ein gutes Beispiel für den Typus dieses "ethnischen entrepreneurs" bietet der singhalesische Politiker S.W.R.D. Bandaranaike. Er gehört nicht der alten singhalesischen Elite an, er hat lediglich in sie eingeheiratet. Die hohen Positionen innerhalb der alten, westlich orientierten und größten Partei des Landes, der UNP, bleiben ihm verstellt. 19151 tritt er aus dieser United National Party aus und begründet die programmatisch Sri Lanka Freedom Party, SLFP, genannte Partei. Sie trägt die singhalesische Bezeichnung Ceylons und sie vertritt die Auffassung, daß die Freiheit des Landes für die singhalesische Mehrheit erst noch politisch zu erringen sei. 
ansprechen, die sich nun mühelos in die politische Sphäre verlängern läßt, können sie ihren Einfluß auch gegenüber den formal nicht-politischen Angelegenheiten im Binnenraum ihrer Wählergemeinschaft geltend machen. Entweder waren oder werden sie nun die traditionellen Meinungsführer, die Autoritäten in ihrer ethnischen Gemeinschaft. Bleibt ihre Führerschaft unbestritten, bilden sie die einzige Partei der betreffenden ethnischen Gruppe, so läßt sich auch die Parteiorganisation auf ein Minimum reduzieren. Bricht sich in Konsequenz ihres Erfolgs ein wachsender Antagonismus zwischen dieser Gruppe und der Gesamtgesellschaft Bahn, so wird diese Entwicklung ihren Anspruch auf Führerschaft eher bestärken. Sie stellen sich schließlich zu Recht als den einzigen und letzten Schutz gegenüber der Feindseligkeit einer Außenwelt dar, die sie vielleicht erst zur Entstehung gebracht haben. Sie können damit gegenüber einer politischen Außenwelt und einer ethnischen Innenwelt ihren Einfluß und ihre Macht steigern.

\section{Schluß}

Die vorhergehende Betrachtung hat gezeigt, daß die Entstehung radikaler und of militanter ethnischer Konflikte drei Bestandteile, Prozesse oder Etappen voraussetzt: Die Relativierung und Idealisierung einer ethnischen Identität, die Bereitschaft und Fähigkeit ökonomische, soziale und kulturelle Konflikte im Lichte dieser emeuerten ethnischen Identität nicht nur zu interpretieren, sondern ethnische Organisationsmuster in diese Konflikte zu tragen, schließlich die Bereitschaft und Fähigkeit, diese einzelnen Konflikte in einen Machtkonflikt zu verwandeln. Diese Politisierung der Konflikte verwandelt ökonomische Ängste, soziale Beschwerden und kulturelle Ressentiments in einen oft schrankenlosen Kampf um politische Macht und Anerkennung.

Keine dieser Etappen leitet sich aber selbstverständlich aus der jeweils vorgängigen ab. Sie bleiben das Resultat besonderer kolonialer und vor allem nachkolonialer Rahmenbedingungen. Sie sind jeweils seltener, aber sie wirken auf die jeweils vorgeschalteten Prozesse wiederum zurück. So gibt die Politisierung und die Radikalisierung dieser Konflikte der ethnischen Elite schließlich die Möglichkeit auf die Rekonstruktion und Idealisierung ethnischer Identität und auf die Interpretation der ethnischen Konfliktlagen einzuwirken und diese Konflikte zu intensivieren. Die Entstehung und Entwicklung der Konflikte fällt damit immer stärker in den Bereich einer sozialen Produktivität, schließlich eines politischen Kalküls. Die Identität und die Interessen der ethnischen Gruppe leiten sich nicht von einem ursprünglichen, einem primordialen Ordnungsentwurf $a b$, sondern von dem Kalkül einer ethnischen Elite. Deren Ambitionen und Ängste bleiben durch die Rahmenbedingungen des modemen Territorialstaates und von der Herausforderung des Modemisierungsprozesses bestimmt. 
Diese ethnischen Konflikte sind damit eher der Gegenwart als der Dritten Welt spezifisch. Sie entstehen in der Schnittlinie von vier Prozessen: Einer umfassenden Relativierung der ethnischen Identität, der raschen Durchsetzung eines zentralisierenden Territorialstaates, einer nachholenden und beschleunigten Modemisierung und Demokratisierung. Lediglich die Intensität, die Schnelligkeit und die Gleichzeitigkeit dieser Prozesse sind dem postkolonialen Staate eigentümlich. 
The policy-makers adopted the pattem provided by existing English law and impliedly rejected the theoretical objections. An interim conclusion is that the view of operative legal systems in which indigenous laws are recognised may be a persuasive factor in overcoming theoretical objections to such recognition.

\title{
Ethnic Conflict in States of the Third World
}

\author{
By Jakob Rösel
}

The following paper explores the emergence of frequent and radical ethnic conflict in the postcolonial states along three perspectives: the colonial heritage, the impact of an accelerated process of modemization and the ambiguous consequences of democratization. Colonial rule preserves and often enhances a delicate system of limited, polyethnic "incorporation into the market place and into the state". After independence processes of economic, cultural, social - modemization of ten weaken, destroy or transform those ethnic "markers" - language, religion, myth of origin, area of settlement - by which different groups traditionally defined themselves. Modemization thus gives rise to a tendency to idealize or even reconstruct a group's ethnic identity and cultural heritage. At the same time, confronted with the challenge of administrative centralization, rapid urbanization and the emergence of central, "national" markets, ethnic groups solidarity now serves the new functions of defending the group's economic interests, of organizing the migration and adjustment to the expanding cities or of securing access to new markets or the growing apparatus of state. It is the transformation of an ethnic lobby into an ethnic voting block or party, that finally leads to the politization and radicalization of ethnic conflicts. Proportionate to the extent that ethnic cleavages become inscribed into the party structure these cleavages can lead to the complete political segmentalization of the electorate and to the dissolution of the precarious democratic framework of the postcolonial state. 